BNL-112112-2016-JA

\title{
Electronic and optical properties of novel carbazole-based donor-acceptor compounds for applications in blue- emitting organic light-emitting diodesce
}

\section{Christian M. Legaspi}

Submitted to the Journal of Physical Chemistry of Interfaces and Nanomaterials XIV

\author{
Christian M. Legaspia, Regan E. Stubbs ${ }^{a}$, David J. Yaron ${ }^{a}$, \\ Linda A. Peteanu ${ }^{a}$, Matthew Y. Sfeir ${ }^{b}$, Abraham Kemboi ${ }^{c}$, \\ Jesse Picker ${ }^{c}$, and Eric Fossum ${ }^{c}$ \\ aDepartment of Chemistry, Carnegie Mellon University, 4400 Fifth Ave., \\ Pittsburgh, PA \\ ${ }^{b}$ Center for Functional Nanomaterials (CFN), Brookhaven National Laboratory, \\ Upton, NY \\ 'Department of Chemistry, Wright State University, 3640 Colonel Glenn Hwy., \\ Dayton, $\mathrm{OH}$
}

Center for Functional Nanomaterials

Brookhaven National Laboratory

\author{
U.S. Department of Energy \\ Office of Basic Energy Sciences
}

Notice: This manuscript has been authored by employees of Brookhaven Science Associates, LLC under Contract No. DE- SC0012704 with the U.S. Department of Energy. The publisher by accepting the manuscript for publication acknowledges that the United States Government retains a non-exclusive, paid-up, irrevocable, world-wide license to publish or reproduce the published form of this manuscript, or allow others to do so, for United States Government purposes. 


\section{DISCLAIMER}

This report was prepared as an account of work sponsored by an agency of the United States Government. Neither the United States Government nor any agency thereof, nor any of their employees, nor any of their contractors, subcontractors, or their employees, makes any warranty, express or implied, or assumes any legal liability or responsibility for the accuracy, completeness, or any third party's use or the results of such use of any information, apparatus, product, or process disclosed, or represents that its use would not infringe privately owned rights. Reference herein to any specific commercial product, process, or service by trade name, trademark, manufacturer, or otherwise, does not necessarily constitute or imply its endorsement, recommendation, or favoring by the United States Government or any agency thereof or its contractors or subcontractors. The views and opinions of authors expressed herein do not necessarily state or reflect those of the United States Government or any agency thereof. 


\title{
Electronic and optical properties of novel carbazole-based donor-acceptor compounds for applications in blue-emitting organic light-emitting diodes
}

\author{
Christian M. Legaspi ${ }^{a}$, Regan E. Stubbs ${ }^{a}$, David J. Yaron ${ }^{a}$, Linda A. Peteanu ${ }^{a}$, Matthew Y. \\ Sfeir $^{b},{\text { Abraham } \text { Kemboi }^{c} \text {, Jesse Picker }}^{c}$, and Eric Fossum ${ }^{c}$ \\ ${ }^{a}$ Department of Chemistry, Carnegie Mellon University, 4400 Fifth Ave., Pittsburgh, PA \\ 15213, USA; \\ ${ }^{b}$ Center for Functional Nanomaterials (CFN), Brookhaven National Laboratory, Upton, NY \\ 11973, USA; \\ ${ }^{c}$ Department of Chemistry, Wright State University, 3640 Colonel Glenn Hwy., Dayton, OH \\ 45435, USA
}

\begin{abstract}
Organic light-emitting diodes (OLEDs) have received a significant attention over the past decade due to their energy-saving potential. We have recently synthesized two novel carbazole-based donor-acceptor compounds and analyzed their optical properties to determine their suitability for use as blue emitters in OLEDs. These compounds show remarkable photo-stability and high quantum yields in the blue region of the spectrum. In addition, they have highly solvatochromic emission. In non-polar solvents, bright, blue-shifted $\left(\lambda_{\max } \approx 398 \mathrm{~nm}\right)$, and highly structured emission is seen. With increasing solvent dielectric constant, the emission becomes weaker, red-shifted $\left(\lambda_{\max } \approx 507 \mathrm{~nm}\right)$, and broad. We aim to determine the underlying cause of these changes. Electronic structure calculations indicate the presence of multiple excited states with comparable oscillator strength. These states are of interest because there are several with charge-transfer (CT) character, and others centered on the donor moiety. We theorize that CT states play a role in the observed changes in emission lineshape and may promote charge mobility for electrofluorescence in OLEDs. In the future, we plan to use Stark spectroscopy to analyze the polarity of excited states and transient absorption spectroscopy to observe the dynamics in the excited state.
\end{abstract}

Keywords: OLEDs, blue-emitters, carbazoles, donor-acceptor compounds

\section{INTRODUCTION}

The need for innovative and viable solutions in the field of renewable energy, particularly for sustainable lighting applications, has motivated the study of fluorescent organic compounds for applications in organic light-emitting diodes (OLEDs). Currently, there are two primary classes of materials used for OLED fabrication: 1) conjugated polymers, and 2) small, fluorescent molecules. For conjugated polymers, the limited control over the polymerization process introduces significant heterogeneity in the system through a distribution of polymer chain lengths and shapes, and the potential for defects in the polymer structure. ${ }^{1}$ This heterogeneity adds significant complexity to these systems, making it difficult to understand and model their structural and electronic properties.

Given the limitations of conjugated polymers, there has been a recent resurgence in research effort toward design of small molecules for OLED applications, both as standalone emitters and as sensitizers. ${ }^{1-6}$ Of particular interest are materials with excellent charge transport properties, ideally for both electrons and holes, as this is crucial to the materials' performance in OLEDs. This has been achieved in OLED systems by layering or blending an electron-transport and a hole-transport material. Recent work has explored the use of intramolecular donor-acceptor (D-A) systems, which tether electron-donating and electron-accepting moieties on the same

Further author information: (Send correspondence to L.A.P.)

L.A.P.: E-mail: peteanu@cmu.edu, Telephone: 14122681327

E.F.: E-mail: eric.fossum@wright.edu, Telephone: 19377752047 
structure. $^{2,7-9}$ This spatially separates the HOMO and LUMO, creating an intramolecular charge-transfer (CT) excited state, allowing a single material to stabilize either a radical cation or anion. Use of one material eliminates the complexities associated with forming multi-layer or blended systems, which is highly advantageous for production. Recent reviews of small molecule donor-acceptors in OLEDs ${ }^{1,2}$ note that there is still a need for efficient blue-emitting systems.

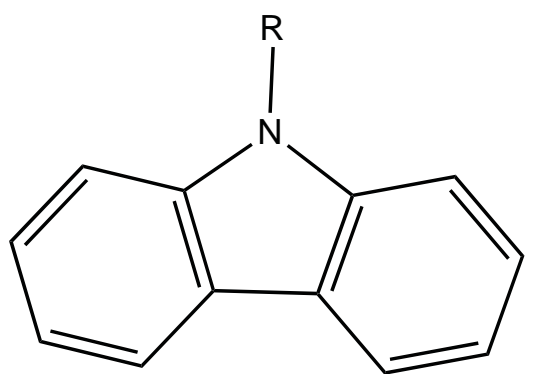

Figure 1: Molecular structure of carbazole $(\mathrm{CBZ}, \mathrm{R}=\mathrm{H})$. For our two compounds of interest, the $\mathrm{R}$ groups are proprietary but have electron-accepting character. These compounds will be referred to as 1-CBZ and 2-CBZ.

Carbazole-based D-A systems have been identified as promising blue CT emitters since carbazole is a weaker electron donor than amine-based donors, widening the HOMO-LUMO gap and blue-shifting emission. ${ }^{2}$ Recent studies by Adachi et al. show that many carbazole compounds undergo a process known as thermally-activated delayed fluorescence (TADF). ${ }^{9-14}$ In TADF, excited compounds in their triplet state undergo reverse intersystem crossing, mediated by the available thermal energy. ${ }^{14}$ This process allows for non-emissive triplets to be recovered as emissive singlets through delayed fluorescence, which generally occurs on the sub-microsecond to the sub-millisecond timescale in deoxygenated solution ${ }^{15,16}$ and in thin films. ${ }^{14,17}$ This is important for overcoming the limitation on electroluminescence quantum yield imposed by spin statistics. ${ }^{14}$ Generally, a small singlet-triplet energy gap $\left(\Delta E_{S T}\right)$ on the order of a few tenths of an eV is necessary for efficient TADF. ${ }^{14}$ However, TADF has been observed in compounds with $\Delta E_{S T}$ as large as $1.1 \mathrm{eV}(\mathrm{kT}$ at $298 \mathrm{~K}$ is $\approx 26 \mathrm{meV}$ ), indicating that singlet-triplet coupling also plays a role in TADF efficiency and that the exact mechanism of TADF is complex. ${ }^{15}$ Carbazole compounds tend to have high triplet energies, and, consequently, a relatively small $\Delta E_{S T}{ }^{2}$ making them ideal candidates for TADF, as well as host materials for triplet transfer in metalcontaining blue phosphorescent OLEDs. ${ }^{18-20}$

Herein, we explore the photoluminescence properties of two novel carbazole-based D-A compounds. The exact structures of these compounds at the time of writing are proprietary. They will be denoted as 1-CBZ and 2-CBZ, where 1 and 2 are proprietary acceptor subunits and CBZ represents the carbazole donor subunit (Figure 1). Steady-state measurements in solution show strong solvatochromic behavior in emission, characteristic of an emissive CT state, which is supported by time-dependent density functional theory (TD-DFT) calculations. In addition, preliminary findings based on transient absorption data show these compounds have long-lived dynamics, a promising indication of TADF.

\section{METHODS}

Synthesis of 1-CBZ and 2-CBZ will not be detailed here, due to the proprietary nature of the molecular structure. Carbazole was purchased from Fisher Scientific and used as received. All solvents used are commercially available from Sigma Aldrich or Fisher Scientific and were purchased at spectroscopic grade or equivalent and used as received. Steady-state absorption and emission measurements were performed using a Cary 50 Bio UV-Vis spectrophotometer and a Jobin-Yvon FluoroMax 2 fluorometer, respectively. UV-transparent quartz fluorescence cuvettes (Starna Cells) with $10.00 \mathrm{~mm}$ pathlengths were used for all measurements.

Theoretical calculations were performed using the Gaussian 09 software package. ${ }^{21}$ Optimized structures and energies were obtained using the CAM-B3LYP functional with the $6-31++\mathrm{G}(\mathrm{d}, \mathrm{p})$ basis set. Solvent effects were included using the polarizable continuum model as implemented for methylcyclohexane. 


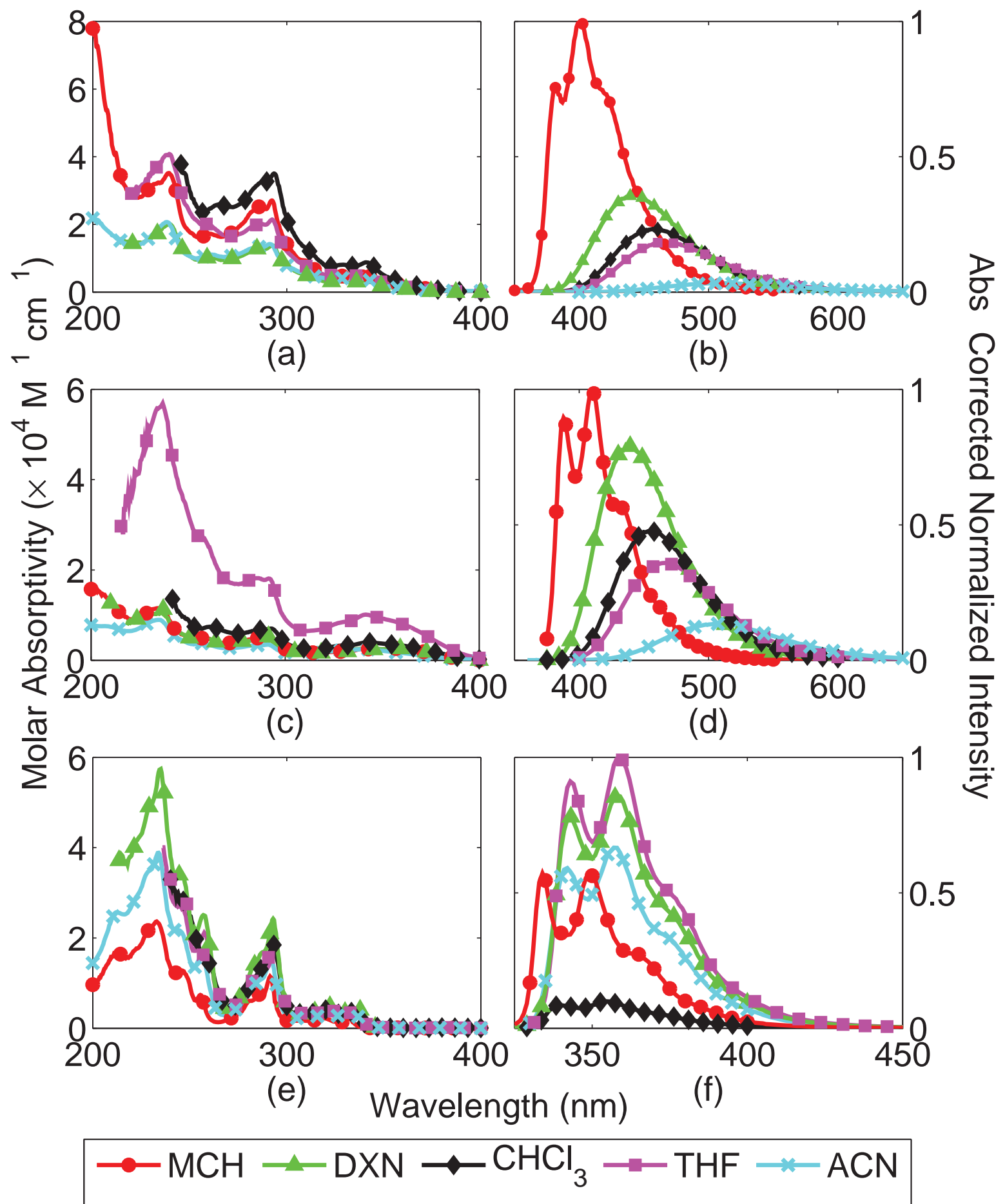

Figure 2: Steady-state absorption (left) and emission (right) for 1-CBZ (a-b), 2-CBZ (c-d), and CBZ (e-f). Emission spectra were collected exciting at the maximum wavelength of the excitation spectra (not shown) in each solvent: 1-CBZ, $\lambda_{\mathrm{ex}} \approx 292 \mathrm{~nm} ; 2$-CBZ, $\lambda_{\mathrm{ex}} \approx 354 \mathrm{~nm}$; CBZ, $\lambda_{\mathrm{ex}} \approx 292 \mathrm{~nm}$. Solvents in order of increasing dielectric constant are methylcyclohexane $(\mathrm{MCH})$, 1,4-dioxane $(\mathrm{DXN})$, chloroform $\left(\mathrm{CHCl}_{3}\right)$, tetrahydrofuran (THF), and acetonitrile (ACN). 


\section{RESULTS AND DISCUSSION}

Steady-state absorption, emission, and excitation spectra were collected for 1-CBZ and 2-CBZ in a variety of solvents with a wide range of polarities (Figure 2a-d). There is no significant variation in peak position in the absorption spectra with solvent polarity, indicating that the Franck-Condon (FC) state is not likely to be polar. A strong, positive solvatochromic trend can be seen in the emission of both compounds, with a range of emission maxima spanning over $100 \mathrm{~nm}$. This is characteristic behavior of a polar emissive state, more specifically a CT state.

Emission intensity notably decreases with increasing solvent polarity. Comparison of the integrated area under the absorbance-corrected emission curves shows that in solvents with broad emission, the relative area decreases with increasing solvent dielectric constant in both samples. This does not hold for the highly-structured emission from the samples in methylcyclohexane $(\mathrm{MCH})$. We surmise that the difference in emission lineshape is a result of a change in electronic character of the emissive state.

By comparison, the CBZ donor alone shows very weak solvatochromism. The absorption (Figure 2e) and emission (Figure 2f) spectra of CBZ show significant lineshape structure in both absorption and emission, indicative of more localized FC and emissive states, like those seen for $\pi \pi^{*}$ transitions. A notable similarity between the D-A compounds and CBZ is that they share many absorption features. This implies that their FC states have similar $\pi \pi^{*}$ character.

\begin{tabular}{cccc}
\hline \hline State & $\Delta E(\mathrm{eV})$ & $\mu(\mathrm{D})$ & $f$ \\
\hline \multicolumn{4}{c}{ 1-CBZ } \\
\hline Ground & - & 1.67 & - \\
1 & 4.23 & 15.4 & 0.42 \\
3 & 4.72 & 6.66 & 0.69 \\
4 & 4.87 & 2.73 & 0.25 \\
6 & 5.09 & 10.4 & 0.30 \\
9 & 5.45 & 1.79 & 0.28 \\
\hline \multicolumn{5}{c}{2 -CBZ } & \\
\hline Ground & - & 2.07 & - \\
1 & 4.09 & 14.8 & 0.22 \\
3 & 4.56 & 4.35 & 0.65 \\
5 & 4.85 & 7.42 & 0.36 \\
6 & 4.87 & 2.66 & 0.27 \\
10 & 5.42 & 1.93 & 0.20 \\
\hline
\end{tabular}

Table 1: Excitation energies $(\Delta E$, in $\mathrm{eV})$, dipole moments ( $\mu$, in Debye), and ground-state oscillator strengths $(f)$ for the 5 states with highest $f$ among the 10 lowest excited singlet states as calculated by TD-DFT in methylcyclohexane for 1-CBZ (top) and 2-CBZ (bottom). Larger dipole moments represent states with significant CT character.

We performed TD-DFT calculations on 1- and 2-CBZ to verify that these compounds do indeed show CT character. Orbital analysis shows that the HOMO resides primarily on the CBZ donor moiety and the LUMO resides on the acceptor moiety. This is characteristic of D-A compounds, and indicates the presence of a CT state. However, calculations also show there are several possible FC states with comparable ground-state oscillator strength (Table 1). As expected, some of these states show primarily CT character, with density moving from CBZ to the acceptor moiety upon excitation, and higher dipole moments. Others, however, have either mixed or primarily $\pi \pi^{*}$ character and are centered on the CBZ moiety with overall lower excited state dipole moments.

The presence of a number of possible FC states with differing character is of particular interest. Emission from excited states with large CT character tends to be broad and unstructured, while $\pi \pi^{*}$ states tend to have more structured emission due to the localized nature of these transitions. As solvent polarity is increased and CT 
states are stabilized, more CT character may be introduced to the emissive state. This state mixing may result in a lineshape change in emission with increasing solvent polarity, similar to that observed. Additionally, an increasingly polar CT emissive state may reduce oscillator strength to the donor-localized ground state, resulting in decreased emission intensity with increasing solvent polarity, like that seen for these D-A compounds. Further investigation is necessary in order to provide a more rigorous explanation of the solvatochromic trends observed and clearer insight into the dynamics of the excited state.

\section{CONCLUSION}

In the current work, we have characterized two novel carbazole-based D-A compounds via steady-state absorption and emission spectroscopies, as well as with TD-DFT calculations. Results indicate strong positive solvatochromism in emission, but not in absorption. This indicates that the nature of the excited state is dynamic, likely involving multiple states with differing character. Calculations show the presence of both CT and $\pi \pi^{*}$-type states with comparable ground-state oscillator strength and similar excitation energies, which may justify the observed solvatochromic behavior.

In the future, we plan to characterize these compounds in films to determine their suitability for use in OLEDs. If film studies prove promising, efforts will be concentrated on designing an OLED from these compounds. As these compounds have CT character, we plan to analyze these compounds using Stark spectroscopy, which is useful for determining the polarity of electronic transitions.

Preliminary work has begun using transient absorption spectroscopy to analyze the dynamics of the excited state. Early results show contributions from multiple excited states, including triplet states. There also appear to be dynamics occurring at very long probe delay times, indicating that thermally-activated delayed fluorescence may be present in these compounds.

\section{ACKNOWLEDGMENTS}

This work was supported by the National Science Foundation Grants CHE-1012529 and CHE-1363050 to LAP, CHE-1027985 to DJY, and CHE-1307117 to EF. Preliminary transient absorption spectroscopy experiments were performed at the Center for Functional Nanomaterials at Brookhaven National Laboratory, which is supported by the U.S. Department of Energy, Office of Basic Energy Sciences, under Contract DE-AC02-98CH10886.

\section{REFERENCES}

[1] Duan, L., Hou, L., Lee, T.-W., Qiao, J., Zhang, D., Dong, G., Wang, L., and Qiu, Y., "Solution processable small molecules for organic light-emitting diodes," J. Mater. Chem. 20(31), 6392 (2010).

[2] Duan, L., Qiao, J., Sun, Y., and Qiu, Y., "Strategies to design bipolar small molecules for OLEDs: Donoracceptor structure and non-donor-acceptor structure," Adv. Mater. 23(9), 1137-1144 (2011).

[3] Reineke, S., Thomschke, M., Lüssem, B., and Leo, K., "White organic light-emitting diodes: Status and perspective," Reviews of Modern Physics 85(3), 1245-1293 (2013).

[4] Yook, K. S. and Lee, J. Y., "Small Molecule Host Materials for Solution Processed Phosphorescent Organic Light-Emitting Diodes," Adv. Mater. 26(25), 4218-4233 (2014).

[5] Yang, X., Xu, X., and Zhou, G., "Recent advances of the emitters for high performance deep-blue organic light-emitting diodes," J. Mater. Chem. C 3(5), 913-944 (2015).

[6] Yu, T., Liu, L., Xie, Z., and Ma, Y., "Progress in small-molecule luminescent materials for organic lightemitting diodes," Sci. China: Chem. 58(6), 907-915 (2015).

[7] Goes, M., Verhoeven, J. W., Hofstraat, H., and Brunner, K., "OLED and PLED Devices Employing Electrogenerated, Intramolecular Charge-Transfer Fluorescence," ChemPhysChem 4(4), 349-358 (2003).

[8] Thirion, D., Rault-Berthelot, J., Vignau, L., and Poriel, C., "Synthesis and Properties of a Blue Bipolar Indenofluorene Emitter Based on a D- $\pi$-A Design," Org. Lett. 13, 4418-4421 (Aug. 2011).

[9] Shizu, K., Lee, J., Tanaka, H., Nomura, H., Yasuda, T., Kaji, H., and Adachi, C., "Highly efficient electroluminescence from purely organic donoracceptor systems," Pure Appl. Chem. 87(7), 627-638 (2015). 
[10] Zhang, Q., Li, J., Shizu, K., Huang, S., Hirata, S., Miyazaki, H., and Adachi, C., "Design of Efficient Thermally Activated Delayed Fluorescence Materials for Pure Blue Organic Light Emitting Diodes," $J$. Am. Chem. Soc. 134(36), 14706-14709 (2012).

[11] Masui, K., Nakanotani, H., and Adachi, C., "Analysis of exciton annihilation in high-efficiency sky-blue organic light-emitting diodes with thermally activated delayed fluorescence," Org. Electron. 14(11), 27212726 (2013).

[12] Ishimatsu, R., Matsunami, S., Shizu, K., Adachi, C., Nakano, K., and Imato, T., "Solvent Effect on Thermally Activated Delayed Fluorescence by 1,2,3,5-Tetrakis(carbazol-9-yl)-4,6-dicyanobenzene," J. Phys. Chem. A 117(27), 5607-5612 (2013).

[13] Wu, S., Aonuma, M., Zhang, Q., Huang, S., Nakagawa, T., Kuwabara, K., and Adachi, C., "High-efficiency deep-blue organic light-emitting diodes based on a thermally activated delayed fluorescence emitter," $J$. Mater. Chem. C 2(3), 421-424 (2014).

[14] Hirata, S., Sakai, Y., Masui, K., Tanaka, H., Lee, S. Y., Nomura, H., Nakamura, N., Yasumatsu, M., Nakanotani, H., Zhang, Q., Shizu, K., Miyazaki, H., and Adachi, C., "Highly efficient blue electroluminescence based on thermally activated delayed fluorescence," Nat. Mater. 14(3), 330-336 (2014).

[15] Dias, F. B., Bourdakos, K. N., Jankus, V., Moss, K. C., Kamtekar, K. T., Bhalla, V., Santos, J., Bryce, M. R., and Monkman, A. P., "Triplet harvesting with $100 \%$ efficiency by way of thermally activated delayed fluorescence in charge transfer OLED emitters," Adv. Mater. 25(27), 3707-3714 (2013).

[16] Lee, D. R., Kim, B. S., Lee, C. W., Im, Y., Yook, K. S., Hwang, S.-H., and Lee, J. Y., "Above $30 \%$ External Quantum Efficiency in Green Delayed Fluorescent Organic Light-Emitting Diodes," ACS Appl. Mater. Interfaces 7(18), 9625-9629 (2015).

[17] Hellerich, E. S., Manna, E., Heise, R., Biswas, R., Shinar, R., and Shinar, J., "Deep blue/ultraviolet microcavity OLEDs based on solution-processed PVK:CBP blends," Org. Electron. 24, 246-253 (2015).

[18] Holmes, R. J., Forrest, S. R., Tung, Y. J., Kwong, R. C., Brown, J. J., Garon, S., and Thompson, M. E., "Blue organic electrophosphorescence using exothermic host-guest energy transfer," Appl. Phys. Lett. 82(15), 2422-2424 (2003).

[19] Jeon, Y. P., Kim, K. S., Lee, K. K., Moon, I. K., Choo, D. C., Lee, J. Y., and Kim, T. W., "Blue phosphorescent organic light-emitting devices based on carbazole/thioxanthene-S,S-dioxide with a high glass transition temperature," J. Mater. Chem. C 3(24), 6192-6199 (2015).

[20] Seo, J.-A., Jeon, S. K., Gong, M. S., Lee, J. Y., Noh, C. H., and Kim, S. H., "Long lifetime blue phosphorescent organic light-emitting diodes with an exciton blocking layer," J. Mater. Chem. C 3(18), 4640-4645 (2015).

[21] Frisch, M. J., Trucks, G. W., Schlegel, H. B., Scuseria, G. E., Robb, M. A., Cheeseman, J. R., Scalmani, G., Barone, V., Mennucci, B., Petersson, G. A., Nakatsuji, H., Caricato, M., Li, X., Hratchian, H. P., Izmaylov, A. F., Bloino, J., Zheng, G., Sonnenberg, J. L., Hada, M., Ehara, M., Toyota, K., Fukuda, R., Hasegawa, J., Ishida, M., Nakajima, T., Honda, Y., Kitao, O., Nakai, H., Vreven, T., Montgomery, Jr., J. A., Peralta, J. E., Ogliaro, F., Bearpark, M., Heyd, J. J., Brothers, E., Kudin, K. N., Staroverov, V. N., Kobayashi, R., Normand, J., Raghavachari, K., Rendell, A., Burant, J. C., Iyengar, S. S., Tomasi, J., Cossi, M., Rega, N., Millam, J. M., Klene, M., Knox, J. E., Cross, J. B., Bakken, V., Adamo, C., Jaramillo, J., Gomperts, R., Stratmann, R. E., Yazyev, O., Austin, A. J., Cammi, R., Pomelli, C., Ochterski, J. W., Martin, R. L., Morokuma, K., Zakrzewski, V. G., Voth, G. A., Salvador, P., Dannenberg, J. J., Dapprich, S., Daniels, A. D., Farkas, ., Foresman, J. B., Ortiz, J. V., Cioslowski, J., and Fox, D. J., "Gaussian09 Revision D.01." Gaussian Inc. Wallingford CT (2009). 